\title{
Informationsverarbeitung und Wirkung von Markenkommunikation
}

Im Rahmen dieser Arbeit geht es um den Einfluss informativen und unterhaltenden Brand Contents auf das Markenimage. Dazu sollen im ersten Teil dieses Kapitels die menschlichen Prozesse bei der Verarbeitung von Markenbotschaften (Informationsverarbeitung) ${ }^{1}$ beleuchtet werden. Dazu wird insbesondere auf Arbeiten aus den Bereichen Persuasions- und Werbewirkungsforschung sowie Psychologie eingegangen. Im zweiten Teil des Kapitels geht es um Priming- und Schema-Effekte. Damit sollen Kongruenzeffekte zwischen Kommunikationskanal, Markenpositionierung und Brand Content erklärt werden.

\subsection{Kognitive und affektive Prozesse der Informationsverarbeitung}

Das individuelle Wissen des Menschen ist in assoziativen Netzwerken gespeichert. Solche Netzwerke oder Schemata bestehen aus Knoten (Wissenseinheit) und Verbindungen zwischen den Knoten (Henderson et al., 1998). In Abschnitt 2.3.3 wurde dargelegt, dass das Markenimage in analoger Weise aus markenbezogenen Assoziationen besteht. Diese Assoziationen können unterschiedlicher inhaltlicher Art sein und sich auf Eigenschaften und Nutzen, aber auch (globale) Einstellungen beziehen (Keller, 1993). Für den Zweck dieser Arbeit wurde das Markenimage in ein funktionales (kognitiv orientiertes) Partialimage, in ein emotionales (affektiv orientiertes) Partialimage sowie in ein Globalimage unterteilt (siehe Abschnitt 2.3.3). Es wurde ebenfalls dargelegt, dass

\footnotetext{
${ }^{1}$ Wenn in diesem Kapitel von Informationsverarbeitung gesprochen wird, dann ist damit die generelle Verarbeitung von Kommunikationsbotschaften gemeint, unabhängig davon, ob diese funktional-informativer oder emotional-unterhaltender Art sind.
} 
Kommunikation eine zentrale Rolle spielt, um markenbezogene Assoziationen bei der Zielgruppe zu verankern und damit das Markenimage zu beeinflussen: Informative Markenbotschaften stärken dabei primär das funktionale Markenimage und emotionale Markenbotschaften primär das emotionale Markenimage (Bruhn, 2014, S. 116-118; Esch, 2019; Keller, 1993; Puto \& Wells, 1984; Ryffel, Wirz, Kühne \& Wirth, 2014). Bei der Verankerung markenbezogener Assoziationen spielen kognitive und affektive Mechanismen eine Rolle.

Die Einstellung zu einem Werbeobjekt kann einerseits auf bestimmten kognitiven Überzeugungen über dieses basieren, die auf Grundlage der durch die Werbebotschaft kommunizierten Objektattribute gebildet werden. (...) Die Einstellung zum Werbeobjekt kann andererseits auf affektiven Informationen basieren, die durch unterschiedliche Mechanismen aus dem Werbekontakt resultieren. (Weber \& Fahr, 2013, S. 339)

Auf diese kommunikativen Prozesse und Mechanismen soll im Rahmen dieses Kapitels eingegangen werden. Dabei wird sich zeigen, dass sowohl beim informativen als auch beim unterhaltenden Brand Content sowohl kognitive und affektive Prozesse laufen als auch das funktionale und das emotionale Markenimage beeinflusst werden können (siehe auch Maio, Haddock \& Verplanken, 2019, S. 152).

Bei Kognition handelt es sich um die «Denk-Dimension» (S. 26) menschlicher Reaktionen auf Kommunikation und Werbung (Vakratsas \& Ambler, 1999). Nach Matthes (2014) umfasst Kognition «die Gesamtheit der informationsverarbeitenden Prozesse und Strukturen eines intelligenten Systems» (S. 13). Diese informationsverarbeitenden Prozesse umfassen mehrere Stufen wie die Wahrnehmung, die gedankliche Auseinandersetzung, die Interpretation oder die Speicherung der Inhalte (Matthes, 2014, S. 13). Wenn sich kognitive Prozesse abspielen, setzen sich Rezipierende mit der Botschaft auseinander, entnehmen dieser gewisse Informationen, speichern diese Informationen ab und erweitern entsprechend das mit der Marke verbundene Wissen (Greenwald, 1968; Maio et al., 2019, S. 121; Rosselli, Skelly \& Mackie, 1995). Der Prozess der Informationsverarbeitung und -speicherung ist dabei selektiv. Es werden nicht alle übertragenen oder vorhandenen Inhalte (z. B. in einer Produktbroschüre) gelernt, sondern nur ausgewählte (Matthes, 2014).

Die Begriffe Affekt und Emotion sind schwierig voneinander abzugrenzen (Poels \& Dewitte, 2019). In Anlehnung an Morris (2012), Schwaiger (2004, S. 49) oder Koeppler (2000, S. 348) werden die Begriffe Affekt und Emotion in dieser Arbeit synonym benutzt. Bei Affekt oder Emotion handelt es sich um die «Gefühls-Dimension» (Vakratsas \& Ambler, 1999, S. 26) menschlicher 
Reaktionen auf Kommunikation und Werbung. Es kann zwischen einem zweidimensionalen und einem mehrdimensionalen Affekt- oder Emotionsverständnis unterschieden werden (Petty et al., 2003, S. 752-753). Beim zweidimensionalen Verständnis steht die Valenz, die Einordnung der Emotion auf dem Kontinuum positiv versus negativ, im Zentrum (Petty et al., 2003). Beim mehrdimensionalen Verständnis werden spezifische positive (z. B. Humor, Freude) und negative (z. B. Ekel, Ärger) Emotionen (A. Bartsch, 2014) unterschieden, die sowohl bewusst als auch unbewusst entstehen (Wirth, 2014).

Weber und Fahr (2013, S. 339-340) nennen drei beispielhafte Mechanismen, wie affektive Botschaften wirken: Mere-Exposure-Effekt, evaluatives Konditionieren und Affekt-als-Information (affect-as-information). Gemäss dem MereExposure-Effekt entwickeln Rezipienten allein durch wiederholte Konfrontation eine positive Einstellung gegenüber einem Objekt, ohne das Objekt (im Detail) zu kennen oder Informationen dazu verarbeitet zu haben (Zajonc, 1980). Das evaluative oder affektive Konditionieren ist eine Form des klassischen Konditionierens (Maio et al., 2019, S. 148). Dabei werden Emotionen oder Assoziationen, die zum Beispiel durch eine Werbung oder den Kontext einer Werberezeption hervorgerufen werden, auf die beworbene Marke übertragen (Maio et al., 2019, S. 148). Bei Affekt-als-Information oder Gefühl-als-Information (Schwarz, 2012) fliessen Emotionen, die sich aus dem Kontext der Werberezeption ergeben, in die Beurteilung des Werbeobjekts hinein (Schwarz, 2012).

\subsubsection{Historische Entwicklung}

Unterschiedliche Modelle haben sich mit dem Zusammenspiel und der Wirkung kognitiver und affektiver Prozesse bei der Werbe- und Kommunikationswirkung beschäftigt. Einfache Lerntheorien stehen aus historischer Sicht am Anfang der Wirkungsforschung (Wirth \& Kühne, 2013). Das AIDA-Modell kann als Ausprägung einer solchen Lerntheorie verstanden werden (ebd.). Es bezieht sich insbesondere auf die «Bewerbung» von Produkten. Dem Modell nach muss eine Botschaft zuerst wahrgenommen werden (A: Attention), dann muss der Inhalt der Botschaft Interesse (I: Interest) auslösen und verarbeitet werden. Auf Basis des Inhalts bildet sich eine Einstellung gegenüber dem beworbenen Produkt und womöglich der Wunsch (D: Desire), das Produkt zu kaufen. Dieser Wunsch führt im Idealfall zum tatsächlichen Kauf (A: Action; Kloss, 2012, S. 86).

Nach Kroeber-Riel und Gröppel-Klein (2019, S. 557) handelt es sich beim AIDA-Modell um ein Stufenmodell. Stufenmodelle zeichnen sich dadurch aus, dass Werbewirkungsstufen nacheinander durchschritten werden. Dieser Prozess 
startet mit der Wahrnehmung der Botschaft und führt über deren Verarbeitung zum Kauf der beworbenen Marktleistung (Hüsser, 2016, S. 251). Stufenmodellen ist gemein, dass die Stufen linear, in unveränderter Abfolge durchlaufen werden müssen.

Stufenmodelle basieren grundsätzlich auf der Annahme starker Effekte und passiver Rezipierender (Wirth \& Kühne, 2013, S. 316). Wenn die Botschaft gesehen und «gelernt» wird, dann handelt der oder die Rezipierende gemäss Intention der Botschaft. Entsprechend wurden einfache Lerntheorien und Stufenmodelle kritisiert. Die Kritik richtet sich insbesondere darauf, dass den mentalen Vorgängen des Menschen zu wenig Beachtung geschenkt wird und dass affektive Prozesse stets den kognitiven nachgelagert sind.

Für Petty, Briñol und Priester (2009, S. 130) sind das Sehen und Verstehen einer Botschaft oft notwendige, aber nicht zwingend hinreichende Konditionen für Verhaltensänderungen. Verhaltensänderungen würden zu wesentlichen Teilen auch von individuellen Faktoren beeinflusst werden - Faktoren, die in Stufenmodellen keinen Eingang finden. Die «Cognitive Response Theory» (Greenwald, 1968) leiste hier einen Mehrwert, weil sie den Einfluss der eigenen Gedankengänge bei der Rezeption neuer Information berücksichtigt (Petty \& Briñol, 2012).

Der Rezipient nimmt gemäss der Cognitive Response Theory eine aktive Rolle ein, verarbeitet die neu gewonnenen Informationen zielgerichtet und setzt diese in ein Verhältnis zu seinem bisherigen Wissensstand (Greenwald, 1968). Dabei werden sowohl Pro- als auch Kontraargumente gebildet (Perloff, 2003). Der Einfluss der Kommunikation hängt dabei stärker von den durch die neuen Informationen ausgelösten Gedankengängen ab als von der neuen Information selbst (Greenwald, 1968, S. 149-151). Auch Perloff (2003, S. 121-122) sieht in der Cognitive Response Theory eine Weiterentwicklung der Lerntheorie. Der Mensch wird nicht länger als «schwammartige Kreatur» (Perloff, 2003, S. 122) angesehen, der alle ihm zugetragenen Informationen aufsaugt. Ein Schwachpunkt der Cognitive Response Theory sei allerdings, dass sie von einer hohen mentalen Aktivität des Menschen bei der Informationsverarbeitung - also von einem hohen Involvement - ausgeht (Perloff, 2003).

Hierarchie-Modelle haben diesen Schwachpunkt adressiert, indem sie auch Situationen tiefen Involvements beschreiben. Insbesondere in solchen Situationen können affektive Prozesse den kognitiven Prozessen vorgelagert sein. Ray (1973) präsentierte drei Modelle, die sich darin unterscheiden, in welcher Abfolge bzw. Hierarchie verschiedene Stufen der Informationsverarbeitung stehen. The «Learning Hierarchy» bildet das traditionelle Stufenmodell ab. Bei der «Dissonance-Attribution Hierarchy» kehren sich die Stufen ins Gegenteil um. Der 
Kauf eines Produkts steht am Anfang, danach bildet sich eine Einstellung und schliesslich werden im Sinne eines Lernprozesses wesentliche Attribute gespeichert. Diese Hierarchie findet sich bei involvierten Personen, die sich allerdings mit kaum unterscheidbaren Produktalternativen konfrontiert sehen. Die beiden letzten Schritte dienen im Normalfall dazu, den Kauf zu stärken bzw. zu rechtfertigen. Bei der «Low Involvement Hierarchy» laufen die Stufen wie folgt ab: Kognition - Kauf - Affekt (Einstellung ${ }^{2}$ ). Werbung hilft hier insbesondere dabei, Markenbekanntheit zu etablieren (Kognition), auf die bei der Kaufentscheidung zurückgegriffen wird. Die Einstellungsbildung (Affekt) folgt nach dem Kauf auf Basis der Erfahrungen mit dem Produkt.

Die Berücksichtigung und Bedeutungswahrnehmung des Involvements mündete schliesslich in den Zweiprozessmodellen. Heute werden Einstellungs- oder Imageänderungen - die den Rahmen dieser Arbeit bilden - hauptsächlich mittels Zweiprozessmodellen wie des Elaboration-Likelihood-Modells (ELM) untersucht (Weber \& Fahr, 2013, S. 339). Das ELM vereint primär kognitive Prozesse bei hohem Involvement (z. B. im Sinne der Cognitive Response Theory) und affektive Prozesse bei niedrigem Involvement (Maio et al., 2019, S. 130; Petty \& Wegener, 1999).

Deshalb wird im weiteren Verlauf dieses Kapitels zuerst das ELM vorgestellt. Danach folgen Ausführungen zum Forschungsbereich «Attitude towards ad» und speziell zur Dual-Mediation-Hypothese. Diese befasst sich explizit mit der Wirkung von Werbebotschaften und kann als (partielle) Ergänzung des ELMs angeschaut werden (MacKenzie \& Lutz, 1989). Schliesslich wird auf das von Kroeber-Riel entwickelte Modell der Wirkungspfade eingegangen, das einerseits zwischen hoher und tiefer Aufmerksamkeit und andererseits zwischen informativen und emotionalen Botschaften unterscheidet (Kroeber-Riel \& Gröppel-Klein, 2013). Allen Modellen ist gemein, dass je nach Konstellation von Aufmerksamkeit und Werbebotschaft unterschiedliche Rezeptions- und Beeinflussungsprozesse einsetzen und affektive und kognitive Prozesse eine mehr oder weniger dominante Stellung einnehmen. Dabei ist schliesslich auch zu beachten, dass Werbungen meistens nicht eindeutig der informativen oder emotionalen Kategorie zugeordnet werden können, sondern Aspekte beider Kategorien vereinen (Koeppler, 2000, S. 350).

Abbildung 4.1 gibt einen Überblick über die Einordnung der Modelle der Informationsverarbeitung in den Rahmen dieser Arbeit.

\footnotetext{
${ }^{2}$ Einstellung wird hier als eindimensionale, affektive Einschätzung gemäss dem Erwartungswert-Modell nach Fishbein und Ajzen (1975) verstanden (siehe Abschnitt 2.3.3).
} 


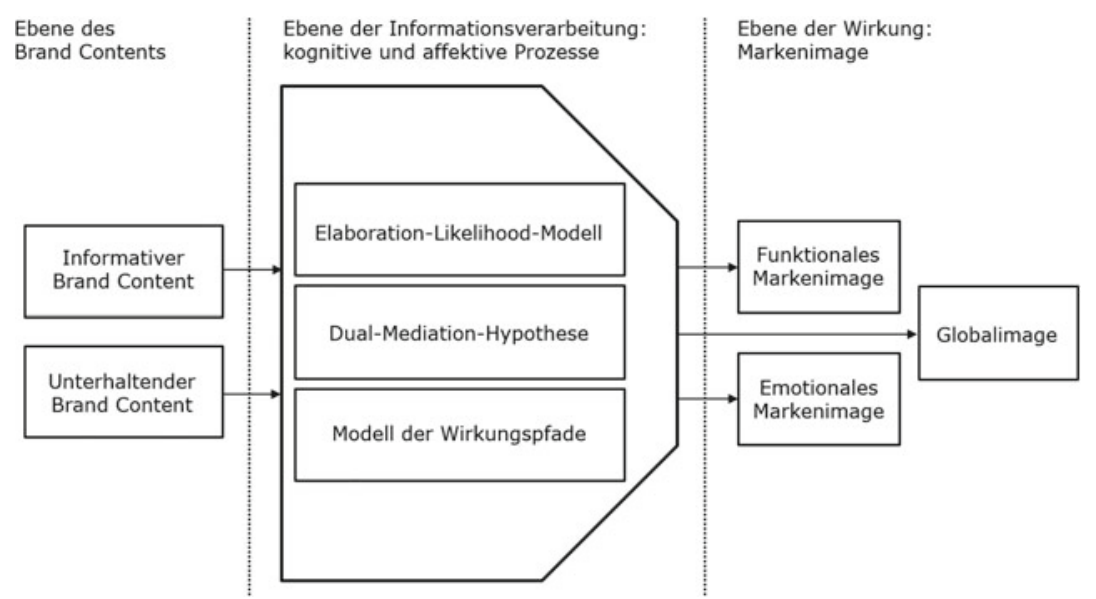

Abbildung 4.1 Einordnung der Informationsverarbeitung im Wirkmodell zu Brand Content. (Eigene Darstellung)

\subsubsection{Elaboration-Likelihood-Modell}

Das Elaboration-Likelihood-Modell geht auf die Arbeiten von Petty und Cacioppo zurück. Die Autoren kritisierten, dass frühere Erklärungsmodelle zu Persuasion und Einstellungsänderungen widersprüchliche Erkenntnisse empirischer Forschung nur ungenügend zu erklären vermochten (Petty, Cacioppo \& Schumann, 1983). Auch weil ignoriert wurde, dass Informationen auf verschiedene Weisen rezipiert werden können: «people are neither universally thoughtful in evaluating persuasive messages nor universally mindless» (Petty \& Cacioppo, 1984, S. 668). Auf diese beiden Enden eines Verarbeitungskontinuums geht das Modell ein, indem es zwei Routen der Informations- oder Botschaftsverarbeitung unterscheidet: eine zentrale und eine periphere Route (Petty \& Cacioppo, 1984, 1986a, 1986b).

Die Informationsverarbeitung über die zentrale Route erfolgt achtsam und mit hohem kognitivem Einsatz. Vorgebrachte Argumente hinsichtlich des Bezugsobjekts (z. B. politische Position, beworbenes Produkt) der Botschaft werden überdacht und aktiv verarbeitet - im Sinne der Cognitive Response Theory (Greenwald, 1968; Petty \& Briñol, 2012). Sowohl Motivation als auch Fähigkeit zur Verarbeitung der Informationen sind hoch. Die Argumentationsqualität der Botschaft spielt entsprechend eine bedeutende Rolle. Einstellungen, die über 
die zentrale Route geändert oder aufgebaut wurden, sind stabil, längerfristig und gute Indikatoren späteren Verhaltens (Petty, Cacioppo, Sedikides \& Strathman, 1988). Auf der peripheren Route sind Motivation und/oder Fähigkeit, Informationen detailliert zu verarbeiten, tief (Petty \& Cacioppo, 1984, S. 668). Es wird deshalb auf sogenannte periphere Hinweise (peripheral cues) zurückgegriffen, um Botschaften einzuschätzen und sich eine Einstellung zu bilden. Beispiele solcher Hinweise können die Attraktivität von Testimonials, die schöne Gestaltung einer Broschüre oder ein Experte als Quelle einer Aussage sein (unabhängig von der tatsächlichen inhaltlichen Qualität). Diese Hinweise dienen in Situationen mit geringer Verarbeitungsmotivation oder -fähigkeit anstelle qualitativ hochwertiger inhaltlicher Argumente der Einschätzung persuasiver Botschaften. Einstellungen, die über die periphere Route entstanden sind, sind weniger persistent und auch ein schlechterer Indikator späteren Verhaltens als Einstellungen, die über die zentrale Route gebildet wurden (Petty et al., 1988). Die Autoren beschreiben die beiden Routen, die in Abbildung 4.2 vereinfacht dargestellt sind, folgendermassen:

The first type of persuasion was that which likely resulted from a person's careful and thoughtful consideration of the true merits of the information presented in support of an advocacy (central route). The other type of persuasion, however, was that which more likely occurred as a result of some simple cue in the persuasion context (e. g., an attractive source) that induced change without necessitating scrutiny of the true merits of the information presented (peripheral route). (Petty \& Cacioppo, 1986b, S. 125)

Eine zentrale Frage, die mit dem ELM einhergeht, ist, wann die Rezipierenden die zentrale Route der Informationsverarbeitung und wann sie die periphere Route durchlaufen (Petty et al., 1983, S. 136). Dem persönlichen Involvement

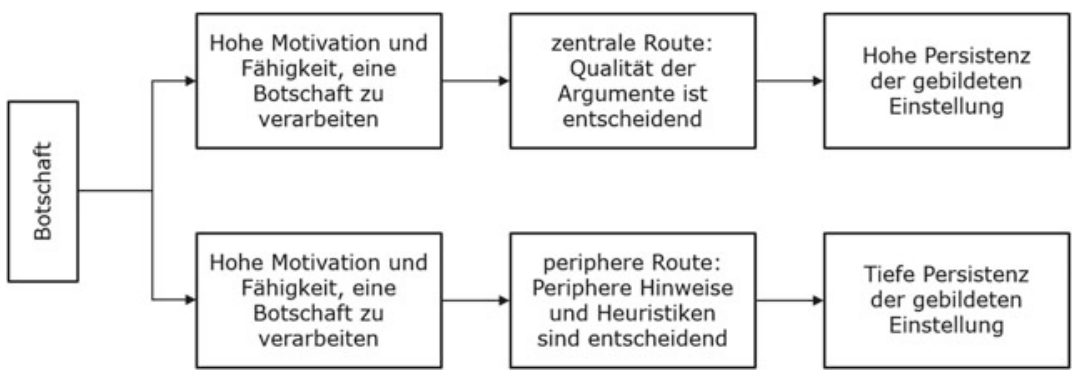

Abbildung 4.2 Elaboration-Likelihood-Modell. (Vereinfachte Darstellung des ELM (Petty \& Cacioppo, 1986b, S. 126) nach Bilandzic, Matthes und Schramm (2015, S. 190)) 
mit dem Bezugsobjekt der Botschaften (z. B. Produkt oder politisches Thema) wird diesbezüglich eine wichtige Rolle zugeschrieben. Mit dem Involvement steigt die Bereitschaft, kognitive Ressourcen zur Informationsverarbeitung einzusetzen, womit auch eine Verarbeitung über die zentrale Route wahrscheinlicher wird (Petty et al., 1983, S. 137).

Die zentrale und die periphere Route bilden die beiden Enden eines Verarbeitungskontinuums ab. Entlang des Kontinuums können verschiedene Prozesse, die sich bezüglich des kognitiven oder affektiven Ausmasses unterscheiden, die Informationsverarbeitung beeinflussen (Petty \& Wegener, 1999). Während die Cognitive Response Theory Verarbeitungsmechanismen nahe dem zentralen Ende abbildet, kommen der Mere-Exposure-Effekt oder evaluatives Konditionieren bei der peripheren Route zum Tragen (Petty \& Briñol, 2012).

Entlang des Kontinuums können Botschaften und deren Elemente unterschiedliche Wirkungen entfalten. Das zeigt sich unter anderem daran, dass eine Variable wie «Schönheit» auf verschiedene Weise auf die Verarbeitung einwirken kann (Petty et al., 1988; Petty \& Wegener, 1999; Petty \& Briñol, 2012):

Wirkung als Argument bei zentraler Verarbeitung: Das Model auf der ShampooVerpackung hat schöne Haare, also werde ich auch schöne Haare bekommen, wenn ich das Shampoo benutze. Die Variable «Schönheit» ist hier eine relevante Information bezüglich des (zu bewerbenden) Produkts und trägt als Argument zur Einstellungsentwicklung bei. Die Variable liefert Informationen «to the central merits of an object or issue» (Petty et al., 1988, S. 359).

Wirkung als «peripheral cue» bei peripherer Verarbeitung: Ein attraktives Model bewirbt einen Kugelschreiber. Schönheit ist hier kein relevantes Argument in Bezug auf die Einschätzung des Produkts, sondern löst beim Betrachter positive Emotionen aus. Schönheit wirkt entsprechend als «peripheral cue [...] in the absence of diligent consideration of the true merits of the object or issue» (Petty et al., 1988, S. 359).

Wirkung als auf die Verarbeitung einwirkende Variable bei mittlerer Verarbeitung: Hier wird die Verarbeitungsmotivation direkt betroffen und durch die einwirkende Variable bestenfalls erhöht. Ein von attraktiven Autorinnen oder Autoren verfasster Artikel erhöht z. B. beim Betrachtenden die Motivation, die im Artikel aufgeworfenen Argumente zu verarbeiten (Petty et al., 1988, S. 361).

Wirkung durch Verzerrung bei zentraler Verarbeitung: Schliesslich können Emotionen, z. B. durch einen schönen Stimulus, bei hohem Involvement die Bearbeitung beeinflussen, indem sie im Sinne der Affekt-Infusion-Hypothese (Forgas, 1995) gewisse Assoziationen hervorrufen bzw. im semantischen Netzwerk aktivieren und die Gedankengänge in Richtung dieser Assoziationen beeinflussen (Petty et al., 2003; Petty \& Briñol, 2012). 
Die ersten drei dieser Wirkweisen besprechen die Autoren (Petty \& Cacioppo, 1984) auch für die Quelle der Botschaft (source factors). Während die Quelle bei hohem Involvement keine Rolle als externer Hinweis spielt, aber ein relevantes Argument hinsichtlich des Bezugsobjekts sein kann, spielt die Quelle bei tiefem Involvement als «peripheral cue» eine tragende Rolle. Bei einem mittleren Involvement schliesslich - wenn der Rezipierende beispielsweise nicht weiss, ob die Information ihn persönlich betrifft, und sich seiner Verarbeitungsmotivation entsprechend ungewiss ist - kann die Quelle als einwirkende Variable die Verarbeitungstiefe beeinflussen. Eine glaubwürdige, relevante oder «attraktive» Quelle steigert die Verarbeitungstiefe Richtung zentrale Route, eine unglaubwürdige, irrelevante, «unattraktive» Quelle mindert die Verarbeitungstiefe Richtung periphere Route. Die Auswirkung auf die Einstellung (ob positiv oder negativ) ergibt sich dann aus der Interaktion mit der inhaltlichen Qualität der zu vermittelnden Botschaft: Eine attraktivere Quelle kann zwar die Verarbeitungsmotivation steigern, beeinflusst aber Einstellungen nicht zwingend positiv. Wird eine Information mit schwachen Argumenten - bei ursprünglich mittlerer Verarbeitungsmotivation - zentral verarbeitet durch den Einfluss einer «attraktiven» Quelle, wirkt sich eine niedrige Argumentqualität negativ auf die Einstellung aus. Umgekehrt wirken sich positive, starke Argumente positiv auf die Einstellung aus, bei erhöhter Verarbeitungstiefe durch den Einfluss einer attraktiven Quelle (Petty \& Cacioppo, 1984).

Es lässt sich festhalten, dass Botschaften und deren Inhalte je nach Involvement unterschiedlich wirken. Die Qualität von Argumenten, die in einer Botschaft aufgeführt werden und für die Beurteilung des Bezugsobjekts relevant sind, hat einen grösseren Einfluss auf die Persuasion über die zentrale Route bei hohem Involvement. Auf der anderen Seite haben periphere Hinweise wie der Expertenstatus einer Quelle, die Atmosphäre während der Informationsverarbeitung oder die Schönheit von Bildern den prägenden Einfluss auf die Persuasion über die periphere Route in Situationen geringen Involvements. Relevante Pro- und Kontraargumente in Bezug auf das Bezugsobjekt der Botschaft spielen eine untergeordnete Rolle und werden - wenn überhaupt - nur mit tiefem kognitivem Einsatz verarbeitet (Petty et al., 1983, S. 135-137; Petty \& Wegener, 1999).

Allerdings können auch Merkmale der Botschaft einen Einfluss auf die Verarbeitungsstrategie haben: Nach Johar und Sirgy (1991) werden utilitaristische (i. S. v. informativ) Botschaften primär über die zentrale Route und transformative (i. S. v. emotional) Botschaften primär über die periphere Route verarbeitet. Bei der Verarbeitung utilitaristischer Botschaften wirkt die sogenannte funktionale Kongruität. Diese liegt vor, wenn die funktionalen Leistungsattribute (product utilitarian attributes, S. 26) einer Marke (z. B. ist leicht zu tragen) mit den durch 
den Rezipienten vorgesehenen Referenzattributen (referent attributes, S. 26) übereinstimmen. Wenn funktionale Kongruität spielt, dann müssen die Argumente in den Botschaften präzise verarbeitet werden. Das geschieht über die zentrale Route im ELM (Johar \& Sirgy, 1991, S. 27). Bei transformativen Botschaften wirkt hingegen die «Selbst-Kongruität». Diese liegt vor, wenn das vom Rezipienten einer Markenbotschaft wahrgenommene prototypische Markennutzer-Image mit dem eigenen Image übereinstimmt (Johar \& Sirgy, 1991, S. 24). Bei Verarbeitungsprozessen über «Selbst-Kongruität» liegt der Fokus auf «source cues», die mit dem eigenen Image in Übereinstimmung gebracht werden. Entsprechend kann Verarbeitung über «Selbst-Kongruität» als «eine Form der peripheren Verarbeitung» (S. 27) angeschaut werden (Johar \& Sirgy, 1991). ${ }^{3}$

Ein weit verbreitetes Gestaltungsmerkmal emotional-unterhaltender Kommunikation ist Humor (Knop, 2006). Humor kann, das wurde in Abschnitt 3.9.2 ausgeführt, als Teil eines emotional-hedonistischen (Unterhaltungs-)Erlebnisses verstanden werden (A. Bartsch, 2014; Oliver \& Raney, 2011). Der Verarbeitung von Humor liegen sowohl kognitive als auch affektive Prozesse zugrunde (Eisend \& Kuss, 2018). In der Literatur wird Humor als Auflösung einer «Schema-Inkongruenz» (S. 35) verstanden, welche kognitive Ressourcen beansprucht und im Erfolgsfall affektive Reaktionen wie Freude hervorruft (Strick, van Baaren, Holland \& van Knippenberg, 2009). Wenn die Auflösung der «AnomalieErfahrung» (S. 345), so die Charakterisierung von Humor nach Eisend und Kuss (2018), in hohem Masse kognitive Ressourcen beansprucht, wirkt sich das negativ auf die Verarbeitung des eigentlichen Botschaftinhalts aus (Eisend \& Kuss, 2018). Das ist insbesondere problematisch, wenn der Humor in keinem Zusammenhang mit der eigentlichen Botschaft, z. B. einem beworbenen Produkt, steht (unrelated humor) und damit nicht die «true merits» des Bezugsobjekts im Sinne des ELM betrifft (Strick et al., 2009). Zudem kann sich die Motivation, eine Botschaft im Detail zu verarbeiten, verringern, wenn sich Menschen in einer durch Humor induzierten guten Stimmung befinden (Martin \& Ford, 2018, S. 206). Beide Fälle führen dazu, dass die eigentliche Botschaft nur in reduzierter Weise und über die periphere Route verarbeitet wird (Martin \& Ford, 2018, S. 207). Humor ohne

\footnotetext{
${ }^{3}$ Johar und Sirgy (1991) schreiben weiter, dass Botschaften dann effektiver wirken, wenn ihre Ausrichtung (utilitaristisch versus transformativ) mit der Ausrichtung (utilitaristisch versus transformativ) des Bezugsobjekts der Kommunikation übereinstimmt. Verfügt eine Marke über eine utilitaristische Positionierung, dann eignen sich utilitaristische Botschaften besser, diese Marke zu bewerben. Diese Überlegungen stimmen mit denjenigen überein, die auch Vaughn (1986) oder Rossiter et al. (1991) in ihren Modellen postuliert haben (siehe Abschnitt 2.3.5).
} 
Bezug zum Werbeobjekt wirkt schliesslich oft im Sinne einer affektiven Konditionierung, wobei die positiven Emotionen des Stimulus auf die Marke übertragen werden (Strick et al., 2009). Insgesamt wirkt Humor deshalb ebenfalls primär über die periphere Route (Martin \& Ford, 2018, S. 205; Strick et al., 2009; Y. Zhang \& Zinkhan, 2006).

\subsubsection{Einstellung gegenüber der Werbung: Dual-Mediation-Hypothese}

$\mathrm{Ab}$ den 1980er-Jahren wurde attitude toward the ad $\left(A_{a d}\right) \mathrm{zu}$ einem zentralen Thema in der Marketing- und Werbeforschung (Muehling \& McCann, 1993). Die Forschung entstand vor dem Hintergrund, dass spontane, affektive Reaktionen auf eine Werbung die Einstellung gegenüber der beworbenen Marke beeinflussen können - ohne dass im Sinne einer kognitionsdominierten Verarbeitung zuerst die Auffassungen (beliefs) der Marke neu beurteilt werden (Muehling \& McCann, 1993). Die Erkenntnis, wonach $A_{a d}$ in der Wirkungskette «Werbung - Einstellung zur Marke - Kaufabsicht» ein wichtiges, intervenierendes Konstrukt ist, war auch für die Werbeindustrie von grosser Bedeutung (Burton \& Lichtenstein, 1988; MacKenzie \& Lutz, 1989).

MacKenzie und Lutz (1989) definierten $\mathrm{A}_{\mathrm{ad}}$ «as a predisposition to respond in a favorable or unfavorable manner to a particular advertising stimulus during a particular exposure occasion» (S. 49). $\mathrm{A}_{\mathrm{ad}}$ wird dabei als affektive Einstellung gegenüber einer spezifischen, rezipierten Werbung direkt nach der Rezeption dieser Werbung verstanden (MacKenzie et al., 1986, S. 130; MacKenzie \& Lutz, 1989, S. 49). In Abschnitt 2.3 .3 wurde bereits dargelegt, dass Einstellungen je nach Auffassung eindimensional (v. a. affektiver Art) oder mehrdimensional konzipiert werden. Entsprechend stiess auch die eindimensionale, affektive Konzeption von $A_{a d}$ wiederholt auf Kritik (Koeppler, 2000, S. 366). Von anderen Forschenden wurde $\mathrm{A}_{\mathrm{ad}}$ zweidimensional als affektive und kognitive Beurteilung (Burton \& Lichtenstein, 1988) oder mehrdimensional (Olney, Holbrook \& Batra, 1991) gemessen. Zudem spielen affektive und kognitive Komponenten bei der Beurteilung der Werbung - als Antezedenzien von $\mathrm{A}_{\mathrm{ad}}$ - eine Rolle (MacKenzie \& Lutz, 1989). Faktoren wie Humor oder bekannte Persönlichkeiten als Testimonials (Muehling \& McCann, 1993), die durch die Werbung ausgelösten Gefühle (Edell \& Burke, 1987), die generelle Einstellung gegenüber Werbung oder die Glaubwürdigkeit der Werbung (MacKenzie \& Lutz, 1989) können sich auf $\mathrm{A}_{\mathrm{ad}}$ auswirken. 
Die Ursprünge des Konzepts gehen auf die Artikel von A. A. Mitchell und Olson (1981) und Shimp (1981) zurück (Muehling \& McCann, 1993). A. A. Mitchell und Olson (1981) interessierten sich vor dem Hintergrund von Fishbein und Ajzens Modell (1975) dafür, ob Auffassungen (product attribute beliefs, S. 320) bezüglich einer Marke die einzigen Determinanten der Einstellung gegenüber einer Marke sind. In einer experimentellen Werbewirkungsstudie für Kosmetiktücher verglichen sie vier verschiedene Stimuli für jeweils vier verschiedene fiktive Marken. In einer der vier Werbungen wurde verbal auf das Attribut «Sanftheit» hingewiesen ohne Verwendung eines Bildes. Die drei anderen Werbungen zeigten ein Bild (Kätzchen, Sonnenuntergang, Gemälde) zusammen mit dem fiktiven Markennamen ohne verbale Nennung eines Attributs. Im Nachgang an die Präsentation der Stimuli mussten die Teilnehmer Produktattribute, die Einstellung gegenüber der Werbung und die Einstellung gegenüber der Marke einschätzen. Die Resultate zeigen, dass sich die Einstellung gegenüber der Marke nicht allein durch die Produktattribute (bzw. product attribute beliefs) ergibt, sondern auch durch die Einstellung gegenüber der Werbung. Die Autoren schlussfolgerten, dass die gleichzeitige Darstellung einer unbekannten Marke in einem Werbemittel mit einem positiv assoziierten Werbebild im Sinne einer klassischen Konditionierung zu einer positiven Einstellung gegenüber der Marke führen kann. In Situationen, in denen die marken- oder produktrelevanten Informationen des Werbematerials nicht verarbeitet werden, kann die Einstellung gegenüber der Werbung die Einstellung gegenüber der Marke positiv beeinflussen (A. A. Mitchell \& Olson, 1981).

Shimp (1981) geht in seinem Artikel zuerst auf «attitude toward the brand» (hier abgekürzt als $A_{b}$; im Originalartikel als $A_{T T b}$ ) ein. Dem Ansatz $A_{b}$ liegt die Überlegung zugrunde, dass Werbung über die Vermittlung von Produktfunktionalität und Produktnutzen ein positives Bild der Konsequenzen eines Produktkonsums zeichnet und damit die Einschätzung der Kundinnen und Kunden einer Marke positiv beeinflusst. Werbung dient hier primär zur Befriedigung von «consumer problems» (S. 9). Dieser Ansatz fruchte aber, so der Autor, nur so lange, wie die beworbene Marke einen echten Mehrwert im Vergleich zu Konkurrenzprodukten biete. Dort, wo dieser Mehrwert wegfällt, kommt $\mathrm{A}_{\mathrm{ad}}$ zum Tragen. Bei $A_{a d}$ geht es nicht länger um spezifische Produktattribute und um die Vermittlung eines positiven Markenbildes. Vielmehr soll die Werbung beim Betrachtenden ein gutes Gefühl auslösen (Shimp, 1981). Während sich $A_{b} a m$ rationalen Konsumierenden orientiert, zielt $\mathrm{A}_{\mathrm{ad}}$ auf hedonistische Motive und eine geringere Verarbeitungstiefe. Shimp präsentiert drei verschiedene Pfade, entlang derer die Markenwahl beeinflusst werden kann. Der erste Pfad orientiert sich an einem klassischen Lernpfad und erfordert hohes Kundeninvolvement. Durch eine 
ausgeprägte Auseinandersetzung mit der Werbung und deren Argumenten bildet sich der Konsumierende eine Einstellung gegenüber der Marke. Der dritte Pfad widmet sich explizit Situationen am Point of Sale (POS). Konsumierende kommen hier nicht mit Werbung in Berührung, sondern die Markenwahl geschieht anhand des am POS vorhandenen Markenmaterials. Beim zweiten Pfad bedient sich der Kunde oder die Kundin sogenannter Heuristiken, also einfacher Entscheidungsregeln, um zwischen Markenalternativen zu wählen. Neben früheren Erfahrungen mit Produktalternativen als eine mögliche Regel kommt hier insbesondere der Einfluss von $A_{a d}$ als Entscheidungsregel zum Tragen. Der Kunde oder die Kundin «may simply transfer his feeling for the ad to the brand» (Shimp, 1981, S. 12). Die mit der Werbung verbundenen Gefühle werden auf die Marken transferiert und dienen damit der Markenwahl.

Unter anderem durch diese beiden Artikel wurde eine rege Forschungstätigkeit über die Einstellung zur Werbung angestossen. Den Höhepunkt erreichte das Thema Ende der 1980er-Jahre. In diese Zeit fallen auch wegweisende Artikel von MacKenzie et al. (1986) und MacKenzie und Lutz (1989). Die Autoren testeten dabei vier verschiedene Modelle über die Einflussnahme von $\mathrm{A}_{\mathrm{ad}}$ (MacKenzie et al., 1986; siehe Abbildung 4.3).

Affect-Transfer-Hypothese

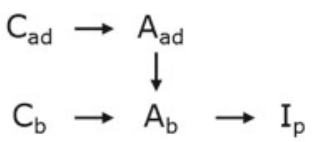

Reciprocal-Mediation-Hypothese

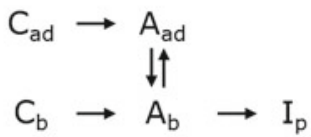

\section{Dual-Mediation-Hypothese}

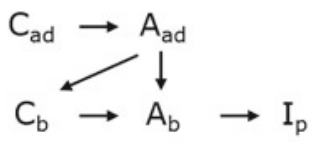

Independent-Influences-Hypothese

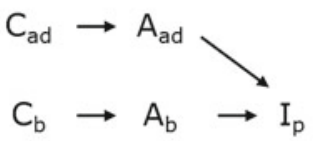

$C_{a d}$ : ad cognitions; $C_{b}$ : brand cognitions; $A_{a d}$ : attitude toward the ad;

$A_{b}$ : attitude toward the brand; $I_{p}$ : intention to purchase the brand

Abbildung 4.3 Vier Hypothesen zur Wirkweise von «Attitude toward the ad». (Nach MacKenzie, Lutz und Belch (1986, S. 131)) 
Drei Wirkungseffekte kommen in allen vier Modellen vor: (1) Werbebezogene Überlegungen und Gedankengänge (Kognitionen, $\mathrm{C}_{\mathrm{ad}}$ ) beeinflussen die Einstellung gegenüber der Werbung $\left(A_{a d}\right)$, (2) markenbezogene Kognitionen $\left(C_{b}\right)$ beeinflussen die Einstellung gegenüber der Marke $\left(A_{b}\right)$ und (3) die Einstellung gegenüber der Marke $\left(A_{b}\right)$ beeinflusst die Kaufintention der Marke $\left(I_{p}\right)$. Damit bildet die Effekthierarchie Kognition - Affekt - Verhalten auch in Bezug auf $\mathrm{A}_{\mathrm{ad}}$ die zentrale Grundlage (Karson \& Fisher, 2005, S. 335; MacKenzie et al., 1986, S. 131).

$A_{a d}$ und $A_{b}$ werden als semantische Differentiale erhoben. $C_{a d}$ als «ad cognitions» und $\mathrm{C}_{\mathrm{b}}$ als «brand cognitions» werden erhoben, indem die Teilnehmenden gebeten werden, ihre Gedanken zur Werbung bzw. zur Marke niederzuschreiben (MacKenzie et al., 1986; Homer, 1990, S. 84). I (intention to purchase) bildet die Kaufintention in Bezug auf das beworbene Produkt ab und wird ebenfalls quantitativ erhoben (MacKenzie et al., 1986, S. 134).

Von den vier getesteten Modellen stellte sich die Dual-Mediation-Hypothese $(D M H)$ als erklärungsstärkstes Modell heraus (MacKenzie et al., 1986). Auch Homer (1990) konnte bei einem Experiment mit einer TV-Werbung und einem Experiment mit einer Print-Werbung den relativen Erklärungsvorteil der DMH gegenüber den anderen drei Alternativen bestätigen. Gemäss der DMH hat die Einstellung gegenüber der Werbung $\left(\mathrm{A}_{\mathrm{ad}}\right)$ einen positiven Einfluss auf die Markenkognitionen $\left(C_{b}\right)$ und auf die Einstellung gegenüber der Marke $\left(A_{b}\right)$.

Die $A_{a d}$-Forschung und insbesondere die damit einhergehende DualMediation-Hypothese können als Ergänzung der Zweiprozessmodelle angesehen werden (Coulter \& Punj, 2004, S. 53; Stenger, 2012, S. 83). Der Link $A_{a d}$ zu $A_{b}$ kann im Sinne der peripheren Route, der Link $C_{b}$ zu $A_{b}$ im Sinne der zentralen Route des ELM verstanden werden (Ahmed, Beard \& Yoon, 2016, S. 134-135; Homer, 1990, S. 79-80; MacKenzie et al., 1986). Der indirekte Link von $A_{a d}$ über $C_{b}$ zu $A_{b}$ besagt ferner, dass die Einstellung gegenüber der Werbung die Neigung des Betrachters, Werbeinhalte zu verarbeiten und zu akzeptieren, beeinflussen kann (Homer, 1990, S. 80). Der indirekte Link kann damit auch im Sinne einer erhöhten Verarbeitungstiefe über eine zentrale Route verstanden werden (Homer, 1990, S. 84).

Zusammenfassend lässt sich auch aus der $\mathrm{A}_{\mathrm{ad}}$-Forschung sagen, dass bei der Betrachtung von Werbewirkungen mindestens zwei Prozesse beachtet werden müssen: einerseits die kognitive Verarbeitung der werblichen Botschaft und die damit verbundenen Einflüsse auf Einstellung und Verhalten (Kognition - Einstellung - Verhalten), andererseits die affektive Reaktion gegenüber der Werbung $\left(\mathrm{A}_{\mathrm{ad}}\right)$ und deren Transfer auf die Einstellung gegenüber der Marke (Huang, Su, Zhou \& Liu, 2013, S. 37). 


\subsubsection{Modell der Wirkungspfade}

Beim Modell der Wirkungspfade nach Kroeber-Riel und Weinberg (2003, S. 612631) spielen sowohl das Involvement der Konsumenten als auch die inhaltliche, emotionale oder informative Art der Werbebotschaft eine zentrale Rolle, um die Werbewirkung zu erklären.

Das Modell basiert auf drei Komponenten (Kroeber-Riel \& Weinberg, 2003, S. 613):

1. Bei den Wirkungskomponenten handelt es sich um die «Bausteine» der Werbewirkung. Dazu gehören der Werbekontakt, die Aufmerksamkeit, kognitive und emotionale Vorgänge, die Einstellung und die Kaufabsicht sowie das Verhalten.

2. Die Wirkungsdeterminanten bestimmen das Zusammenspiel der Wirkungskomponenten. Es werden mit der «Art der Werbung» und dem «Involvement» zwei Determinanten unterschieden.

3. Bei den Wirkungsmustern handelt es sich um «diejenigen Werbewirkungen von (1), die unter den verschiedenen Bedingungen von (2) zu erwarten sind» (S. 613).

Das Modell ist an sich ein hierarchisches Modell, bei welchem «Wechselwirkungen zwischen den Stufen möglich sind»(Kroeber-Riel \& Weinberg, 2003, S. 612). Je nach Kombination von Involvement und Botschaft ergeben sich unterschiedliche Bedingungen der Werbewirkung und damit ein unterschiedlicher Durchlauf durch die Wirkungskomponenten (siehe Abbildung 4.4). Das Involvement wirkt sich beispielsweise auf die Aufmerksamkeit aus: Schwaches Involvement geht mit schwacher Aufmerksamkeit, starkes Involvement mit starker Aufmerksamkeit einher (Kroeber-Riel \& Weinberg, 2003, S. 620).

Eine informative Werbung, die insbesondere «sachliche Informationen» (S. 616) zum Produkt oder zur Marke vermittelt (Kroeber-Riel \& Weinberg, 2003), führt primär zu kognitiven Vorgängen. Eine emotionale Werbung, die auf der «Darbietung emotionaler Reize» (S. 617) basiert, führt primär zu emotionalen Vorgängen.

Kroeber-Riel und Weinberg (2003, S. 616) führen neben der rein informativen und der rein emotionalen auch die gemischte Werbung auf. Durch die Kombination mit Involvement (hoch und tief) ergeben sich sechs «Bedingungen der Werbewirkung». Im Rahmen dieser Arbeit werden die ersten vier Bedingungen näher erläutert (siehe Tabelle 4.1). 
Bedingung 1

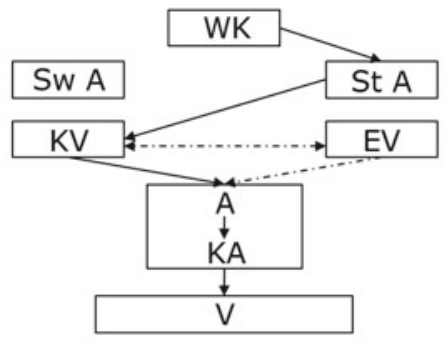

\section{Bedingung 3}

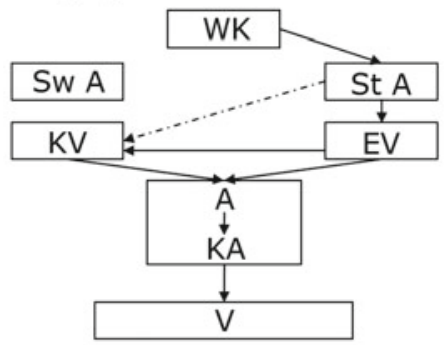

Bedingung 2

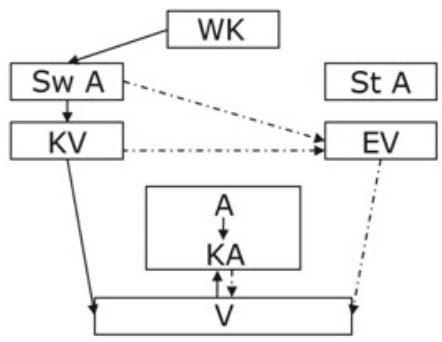

Bedingung 4

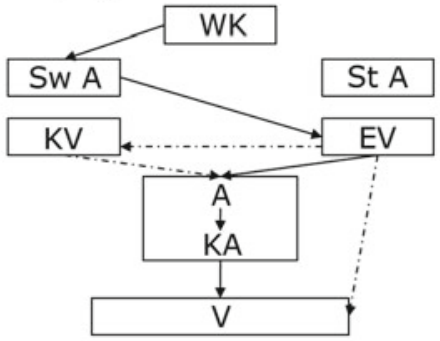

WK: Werbekontakt; Sw A: schwache Aufmerksamkeit; St A: starke Aufmerksamkeit; KV: kognitive Vorgänge; EV: emotionale Vorgänge; A: Einstellung; KA: Kaufabsicht; V: Verhalten

Abbildung 4.4 Modell der Wirkungspfade. (Nach Kroeber-Riel und Weinberg (2003, S. 612-631))

Tabelle 4.1 Bedingungen der Werbewirkung gemäss dem Modell der Wirkungspfade

\begin{tabular}{l|l|l}
\hline & $\begin{array}{l}\text { stark involvierte } \\
\text { Konsumenten }\end{array}$ & $\begin{array}{l}\text { schwach involvierte } \\
\text { Konsumenten }\end{array}$ \\
\hline informative Werbung & Bedingung 1 & Bedingung 2 \\
\hline emotionale Werbung & Bedingung 3 & Bedingung 4 \\
\hline gemischte Werbung & (Bedingung 5) & (Bedingung 6) \\
\hline
\end{tabular}

Anmerkungen. Übernommen aus Kroeber-Riel und Weinberg (2003, S. 616). 


\section{Bedingung 1:}

In Bedingung 1 (Kroeber-Riel \& Weinberg, 2003, S. 622-623) verarbeiten involvierte Konsumenten informative Werbungen. Es dominieren kognitive Vorgänge und eine damit einhergehende umfangreiche Informationsverarbeitung. Die kognitiven Vorgänge werden um «mehr oder weniger starke emotionale Begleitreaktionen [Hervorhebung im Original]» (S. 622) ergänzt (Kroeber-Riel \& Weinberg, 2003), die den Konsumenten aktivieren und sich verstärkend auf die kognitive Informationsverarbeitung auswirken können. Das zeigt sich an den gestrichelten Pfeilen zwischen den beiden Vorgängen. Diese Begleitreaktionen sind dem Involvement der Konsumenten geschuldet. Die Informationsverarbeitung beeinflusst die mit dem Produkt oder der Marke verbundenen Einstellungen und dadurch das Verhalten.

\section{Bedingung 2:}

Unter Bedingung 2 (S. 623-625) ändert sich der Wirkprozess durch das schwache Involvement der Konsumenten. Schwach involvierte Konsumenten schenken der informativen Werbung nur schwache Aufmerksamkeit. Als Folge findet keine umfangreiche Informationsverarbeitung und auch keine Beeinflussung von Einstellungen statt. Insbesondere durch das Wiedererkennen der Marke am POS kann informative Werbung das Verhalten beeinflussen: Der Konsument wählt, was er bereits einmal gesehen hat. Gemäss dem Mere-Exposure-Effekt (Zajonc, 1980) kann sich Sympathie gegenüber einem Produkt bilden, allein dadurch, dass eine Werbung wiederholt gesehen wird. Diese Sympathie kann sich ebenfalls positiv auf das Verhalten auswirken. Eine Einstellungsbildung findet erst nach dem Produktkauf durch die Produktnutzung statt. Der so gezeichnete Wirkungspfad unterscheidet sich von den Überlegungen gemäss dem Elaboration-LikelihoodModell. Über die periphere Route ist dort eine Einstellungsänderung auch bei geringem Involvement möglich (Kroeber-Riel \& Weinberg, 2003, S. 625).

\section{Bedingung 3:}

Unter Bedingung 3 (S. 625-627) werden involvierte Personen mit emotionaler Werbung konfrontiert. Durch das Involvement ergibt sich eine hohe Aufmerksamkeit gegenüber der Werbung. Die Werbung löst primär emotionale Vorgänge aus, die durch das hohe Involvement ihrerseits kognitive Vorgänge anstossen. Das kann auf direkte oder indirekte Weise geschehen. Bei der indirekten Einflussnahme rufen positive durch die emotionale Werbung ausgelöste Gefühle gespeicherte positive Produkteigenschaften hervor. Bei der direkten Einflussnahme werden die «hervorgerufenen emotionalen Eindrücke [werden] direkt mit Produkteigenschaften assoziiert» (Kroeber-Riel \& Weinberg, 2003, S. 626). 
Durch die hohe Aufmerksamkeit ist es möglich, dass selbst der emotionalen Werbung gewisse Informationen (z. B. der Markenname) entnommen werden. Die Verbindung von Aufmerksamkeit hin zu den kognitiven Vorgängen deutet diesen Prozess an.

\section{Bedingung 4:}

Richtet sich schliesslich in Bedingung 4 (S. 627-628) emotionale Werbung an schwach involvierte Konsumenten, dann erklärt sich die Werbewirkung primär anhand der «emotionalen (klassischen) Konditionierung» (S. 627). Über die Werbung «wird eine emotionale Bindung zur Marke hergestellt», welche die Einstellung gegenüber dem Produkt beeinflusst (Kroeber-Riel \& Weinberg, 2003, S. 627). Allerdings ist es auch in dieser Konstellation möglich, dass emotionale Reize direkt zu «Impulskäufen» führen, also das Verhalten beeinflussen, und wenn, dann erst im Nachhinein auf die Einstellung einwirken (S. 628).

\subsection{Priming-Effekte und Schema-Kongruenz}

Im Rahmen dieses Kapitels wird schliesslich auf Priming und Schema-Kongruenz eingegangen, wobei Priming die Grundlage von Schema-Kongruenz bildet. Schema-Kongruenz kann zwischen dem (a) Social-Media-Kanal (als Kontext), (b) der beworbenen Marke und (c) dem Brand Content als Wirkdimensionen der Markenkommunikation auftreten und die Wirkung von Botschaften beeinflussen.

\subsubsection{Priming}

Es wurde bereits beschrieben, dass das mit den Marken verbundene Wissen aus Assoziationen besteht, die in einem semantischen Netzwerk oder Schema miteinander verbunden sind (Henderson et al., 1998). Das gilt nicht nur für Marken, sondern für sämtliche Begriffe oder Objekte.

Priming-Effekte können durch die Schema-Theorie erklärt werden (Schenk, 2007, S. 305): Ein genannter Begriff oder Stimulus (z. B. Fussball) aktiviert den entsprechenden Begriff im semantischen Netzwerk und die mit dem Begriff verbundenen Assoziationen (Ball, Stadion etc.). Je stärker ein Begriff aktiviert wird (z. B. durch längere oder wiederholte Darbietung), desto stärker wird das semantische Netzwerk, in dem der Begriff eingebettet ist, aktiviert bzw. die mit dem 
Begriff verbundenen Assoziationen. Aktivierte Begriffe können, solange die Aktivierung anhält, rascher abgerufen werden als nicht aktivierte Begriffe. Beeinflusst die durch einen vorangegangenen Stimulus hervorgerufene Aktivierung in einem semantischen Netzwerk die Interpretation nachfolgender Informationen, wird von Priming-Effekten gesprochen (Roskos-Ewoldsen et al., 2009, S. 79-81; Schenk, 2007, S. 305-307). Primes lenken entsprechend die Interpretation nachfolgender Inhalte in Richtung der aktivierten Assoziationen. Roskos-Ewoldsen et al. (2009) schreiben: «Priming refers to the effect of some preceding stimulus or event on how we react, broadly defined, to some subsequent stimulus» (S. 74).

Yi (1990b) untersucht anhand von kognitiven Priming-Effekten den Einfluss des Kontextes auf die Interpretation von Aussagen in Werbungen. Er geht davon aus, dass die Interpretation mehrdeutiger Aussagen von den Wissensstrukturen im semantischen Netzwerk abhängig ist, die aktuell aktiviert sind. Ob ein als leicht beworbener Koffer als leicht zu tragen oder als wenig beständig eingestuft wird, hängt unter anderem von aktivierten Wissensstrukturen ab. Der Werbekontext aktiviert als Prime solche Wissensstrukturen, macht gewisse (Produkt-)Attribute zugänglich und beeinflusst damit die Interpretation der Werbung: «The evaluation of the advertised brand would therefore depend on which attribute is activated by the contextual factors preceding or accompanying the ad»(Yi, 1990b, S. 216). Ebenso wie kognitive Priming-Effekte können auch affektive Priming-Effekte die Interpretation beeinflussen. Positive oder negative durch den Kontext ausgelöste Gefühle wirken dann auf die Wahrnehmung der Marke ein (Yi, 1990a, 1990b).

Im Rahmen dieser Arbeit spielen durch digitale Kanäle und Marken ausgelöste Priming-Effekte eine Rolle. Beim Priming durch Marken wird davon ausgegangen, dass das mit der Marke verbundene, aktivierte Wissen die Wirkung des Brand Contents beeinflusst. Beim Priming durch Kanäle wird angenommen, dass die mit dem Kanal verbundenen Gratifikationserwartungen (als eine Form aktivierten Wissens) die Beurteilung von Brand Content beeinflussen. Auf Basis von Erfahrungen hat man gewisse Erwartungen an einen Kontext. So erwartet man beispielsweise in einer Sportzeitung Sportnachrichten und in einer Wirtschaftszeitung Wirtschaftsnachrichten. Die Wahrnehmung des Kontexts aktiviert dabei die entsprechenden Schemata, und diese werden für die Verarbeitung der Inhalte (u. a. Werbung) übernommen. 


\subsubsection{Schema-Kongruenz}

Im Gegensatz zur Arbeit von Yi (1990) geht es im Rahmen dieser Arbeit nicht darum, inwiefern einzelne Wörter auf Basis des Primes interpretiert werden, sondern inwiefern sich thematische Kongruenz zwischen einem Prime und einer nachfolgenden Botschaft auf die Beurteilung der Botschaft auswirkt (Pelsmacker, Geuens \& Anckaert, 2002; Segev, Wang \& Fernandes, 2014). In diesem Zusammenhang wird auch von Schema-Kongruenz gesprochen. Schema-Kongruenz bedeutet, dass die durch einen Kontext aktivierten Schemata mit dem Stimulus übereinstimmen (S. S. Wang et al., 2018, S. 74). Eine Werbung wird einfacher verarbeitet, wenn diese in einem kongruenten Kontext gespielt wird. Der Kontext aktiviert dabei gewisse Assoziationen oder Wissensstrukturen, auf die bei der Verarbeitung der Werbung wieder zurückgegriffen werden kann (Pelsmacker et al., 2002). Aktivierte Schemata entlasten die Informationsverarbeitung. Botschaften müssen nicht von null auf interpretiert werden, weil die aktivierten Schemata der Botschaft einen Rahmen geben und deren Interpretation strukturieren (Matthes, 2014, S. 20). Diese unterstützte Informationsverarbeitung führt zu einer erhöhten Verarbeitungsflüssigkeit (Processing Fluency; Alter \& Oppenheimer, 2009; Jiang, Guan \& Haaij, 2019), die sich schliesslich positiv auf die Einstellungsbildung auswirkt (Estes, Gibbert, Guest \& Mazursky, 2012; Jeong \& King, 2010). Auch affektive, kontextbasierte Primes können die Wahrnehmung von Botschaften im Sinne von Kongruenz beeinflussen (Pelsmacker et al., 2002). Wenn die von der Werbung vermittelten Gefühle den Gemütszustand des Rezipienten, unter anderem ausgelöst durch den Kanal, abbilden, kann dies die Verarbeitung der Werbung erleichtern und zu einer besseren Bewertung von dieser führen (Pelsmacker et al., 2002, S. 50).

Einen Bezug zu dieser Art von Priming-Effekten weist die ResourceMatching-Theorie auf (Coulter \& Punj, 2004; Meyers-Levy \& Peracchio, 1995). Diese besagt, dass die Wirkung einer Botschaft am grössten ist, wenn die für die Rezeption der Botschaft notwendigen kognitiven Ressourcen $(\mathrm{RR}=$ resources required) gleich sind den vom Rezipienten eingesetzten kognitiven Ressourcen $(\mathrm{RA}=$ resources available). Werden entsprechend Menschen durch ein Medium auf Unterhaltung «geprimt», dann ist ihre Motivation tief, kognitiv anstrengende Botschaften zu verarbeiten. Dieser Umstand führt zu einer oberflächlichen Verarbeitung bzw. zu einer starken Bezugnahme auf periphere Hinweise. Werden Menschen durch ein Medium auf Information «geprimt», dann sind sie eher bereit, kognitive Ressourcen für die Verarbeitung einer Botschaft einzusetzen. 
Absorbiert allerdings der Kontext in signifikanter Weise die kognitiven Ressourcen, bleiben ungenügend Ressourcen zur Verarbeitung einer Botschaft (Coulter \& Punj, 2004; Meyers-Levy \& Peracchio, 1995).

In verschiedenen Untersuchungen wurden allerdings auch negative Konsequenzen bezüglich einer Kongruenz zwischen Kontext und Werbung eruiert. Durch «kognitive Interferenz» (Furnham, Bergland \& Gunter, 2002) verschlechtert sich beispielsweise die Erinnerungsleistung an eine Werbung, wenn diese in einem kongruenten Kontext gespielt wird. Die Unterscheidung zwischen Kontext und Werbung, so die Theorie, wird schwieriger und die Elemente des Kontextes und der Botschaft beginnen sich zu vermischen (Furnham et al., 2002). Bei Inkongruenz zwischen Kontext und Werbung kann hingegen nicht in erwartetem Masse auf die mit dem Kontext verbundenen Schemata zurückgegriffen werden. In unterschiedlichen Studien führte Inkongruenz zu erhöhter Verarbeitungstiefe und einem besseren Erinnerungsvermögen an die Werbung (Jeong \& King, 2010, S. 251-252; Törn \& Dahlén, 2007).

Während Inkongruenz insbesondere zu positiven Effekten auf die Erinnerungsleistung führt, werden auch moderater Inkongruenz positive Effekte auf die Beurteilung von Botschaften zugesprochen, sofern die Inkongruenz aufgelöst werden kann (S. S. Wang et al., 2018, S. 75). Zudem wird dem Involvement des Rezipienten eine intervenierende Wirkung bezüglich Vorzug von Kongruenz oder Inkongruenz zugeschrieben - wobei auch hier die Erkenntnisse nicht eindeutig sind (Pelsmacker et al., 2002; Segev et al., 2014).

Open Access Dieses Kapitel wird unter der Creative Commons Namensnennung 4.0 International Lizenz (http://creativecommons.org/licenses/by/4.0/deed.de) veröffentlicht, welche die Nutzung, Vervielfältigung, Bearbeitung, Verbreitung und Wiedergabe in jeglichem Medium und Format erlaubt, sofern Sie den/die ursprünglichen Autor(en) und die Quelle ordnungsgemäß nennen, einen Link zur Creative Commons Lizenz beifügen und angeben, ob Änderungen vorgenommen wurden.

Die in diesem Kapitel enthaltenen Bilder und sonstiges Drittmaterial unterliegen ebenfalls der genannten Creative Commons Lizenz, sofern sich aus der Abbildungslegende nichts anderes ergibt. Sofern das betreffende Material nicht unter der genannten Creative Commons Lizenz steht und die betreffende Handlung nicht nach gesetzlichen Vorschriften erlaubt ist, ist für die oben aufgeführten Weiterverwendungen des Materials die Einwilligung des jeweiligen Rechteinhabers einzuholen.

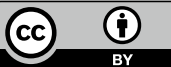

